\title{
Intelligentes Condition Monitoring von hydraulischen Anlagen
}

\author{
Nikolai Helwig ${ }^{1}$, Andreas Schütze ${ }^{1,2}$ \\ 'Zentrum für Mechatronik und Automatisierungstechnik gGmbH, 66121 Saarbrücken \\ ${ }^{2}$ Lehrstuhl für Messtechnik, Universität des Saarlandes, 66123 Saarbrücken \\ Tel.: 068185787 41, e-mail: n.helwig@mechatronikzentrum.de
}

\section{Kurzfassung}

In diesem Beitrag wird eine Zustandsüberwachung für Hydraulikaggregate vorgestellt, welche aus einer Vielzahl von Sensordatenströmen automatisiert Merkmale extrahiert und diese mittels Korrelationsanalyse auf die Eignung zur Detektion unterschiedlicher Schadenszustände überprüft. Die jeweils signifikantesten Merkmale werden zum Training einer linearen Diskriminanzanalyse genutzt und anhand der Diskriminanzfunktionen klassifiziert. Das Verfahren wurde auf eine hydraulische Anlage angewendet und erfolgreich validiert. Des Weiteren wurde untersucht, inwieweit der Ausfall einzelner Sensoren von den übrigen funktionsfähigen Sensoren kompensiert werden kann.

\section{Einleitung}

Das Condition Monitoring (CM) von hydraulischen Anlagen zur Minimierung von Maschinenstillstandszeiten und Optimierung von Wartungsarbeiten findet neben dem Windenergiesektor [1,2] auch in der Industrie und mobilen Arbeitsmaschinen zunehmend Verwendung. Am Markt etablierte CM-Systeme beurteilen den Anlagenzustand meist in der Zeit- oder Frequenzdomäne anhand einer Schwellwertuntersuchung der einzelnen Sensordatenströme. Daneben finden sich in der Literatur verschiedene multivariate Ansätze zur Zustandsüberwachung von hydraulischen Anlagen, die auf künstlichen neuronalen Netzen [3], Entscheidungsbäumen [4] und semantisch-statistischer Analyse [5] basieren. In der vorliegenden Arbeit soll ein statistisches Verfahren gezeigt werden, das für 
eine automatisierte Auswertung geeignet und mit geringem Aufwand auf geänderte Rahmenbedingungen (Ausfall eines Sensors, Auftreten eines neuen Schadensfalls, Änderung der Anlagenkonfiguration) adaptierbar ist.

\section{Konzept}

Die Analyse der Sensordaten erfolgt in zwei Stufen (Bild 1): Zunächst wird die Zustandsüberwachung offline anhand von historischen Daten mit bekannter Klassifikation trainiert. Daraufhin ist das System in der Lage, den aktuellen unbekannten Zustand der Anlage online zu klassifizieren.

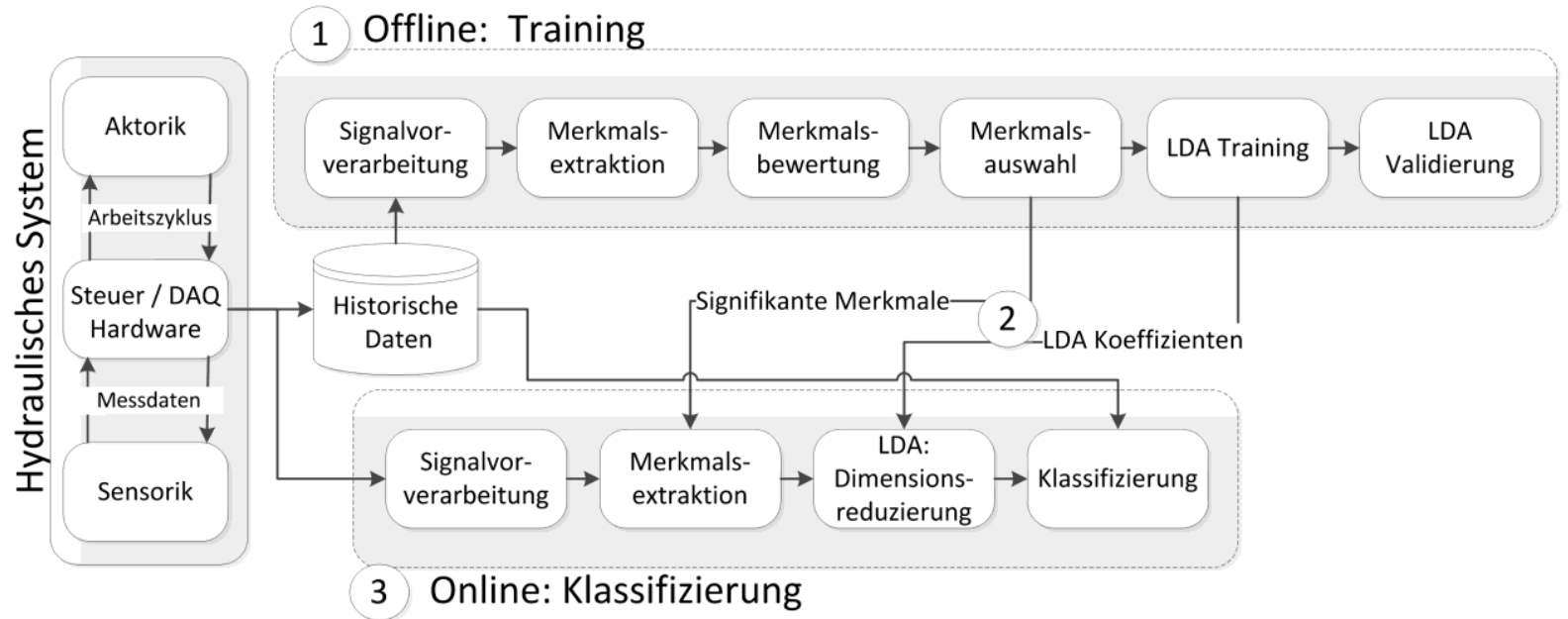

Bild 1: Konzept der Analyse: (1) Trainingsphase, (2) Übergabe der Parameter, (3) Durchführung der Online-Zustandsüberwachung

Für das Training werden die Sensordaten nach einer Vorselektion der zu berücksichtigenden Arbeitszyklen jeweils in charakteristische Zeitintervalle unterteilt (Bild 3a) und aus diesen Bereichen Merkmale wie arithmetisches Mittel, Standardabweichung und Steigung berechnet. Daraus ergibt sich ein MerkmalsPool, aus denen die aussagekräftigsten Merkmale für den jeweiligen Schadensfall ausgewählt werden müssen. Dies geschieht über die Bestimmung einer Korrelationsmatrix zwischen den zeitlichen Merkmals- und Schadensverläufen, deren Einträge nach Betragsgröße sortiert werden. Die höchstkorrelierenden Merkmale werden zu einem schadensspezifischen Merkmalsvektor zusammengefasst, mit dem eine Lineare Diskriminanzanalyse (LDA, nach Fisher) [6] trai- 
niert wird. Diese reduziert die Dimension des Merkmalsvektors durch lineare Projektion in einen typischerweise ein- oder zweidimensionalen Raum bei Maximierung der Trennung zwischen den Klassen und Minimierung der Trennung innerhalb einer Klasse. Im letzten Schritt erfolgt die Validierung der projizierten Trainingsdaten, z.B. mit einem k-nächste-Nachbarn-Klassifikator. Liefert diese ein zufriedenstellendes Ergebnis, werden die ausgewählten Merkmale und LDA-Koeffizienten zur Online-Klassifizierung verwendet. Tritt während des Online-Betriebes ein neuer Fehlertyp auf, können die entsprechenden Arbeitszyklen zu den Trainingsdaten hinzugefügt und das System neu trainiert werden, sodass der Fehler bei erneutem Auftreten möglichst frühzeitig identifiziert werden kann. Ein weiterer Anwendungsfall ist die Kompensation von defekten Sensoren: Wird ein Sensorausfall detektiert, können die Daten des entsprechenden Sensors aus den Trainingsdaten entfernt und das System mit dem reduzierten Datensatz erneut trainiert werden, da die Chance besteht, dass ein anderer Sensor zumindest teilweise dessen Funktion übernehmen kann.

\section{Experimenteller Aufbau}

Das verwendete Hydraulikaggregat (Bild 2) lässt sich in die Systeme Arbeitskreislauf und Filtrier- und Kühlkreislauf unterteilen, die über einen gemeinsamen Öltank gekoppelt sind. Im Arbeitskreislauf können mit dem ProportionalDruckbegrenzungsventil V2 verschiedene Laststufen für die Hauptpumpe HP1 durchlaufen werden, mit dem Wegeventil V1 wird die Last zugeschaltet oder getrennt. Im Sekundärkreislauf erfolgen die Filtrierung des Öls von Partikelkontamination sowie eine Abkühlung des vom Arbeitskreislauf erhitzten Öls. Das mittels SPS gesteuerte Hydraulikaggregat durchläuft zyklisch verschiedene Ventilschalt- und Lastzustände (Arbeitszyklen, vgl. Bild 3a), welche die üblichen Belastungsvariationen in der Praxis nachbilden. An verschiedenen Punkten des Aggregats werden die Größen Druck, Leistung, Volumenstrom, Vibration und Temperatur gemessen, die Sensorwerte zunächst auf der SPS gebuffert und anschließend zur Speicherung als CSV-Datei zu einem PC via Ethernet übertragen. Mit diesem Vorgehen ist die Echtzeitdatenaufnahme und somit eine gleichbleibende Synchronität innerhalb der Arbeitszyklen gesichert. 


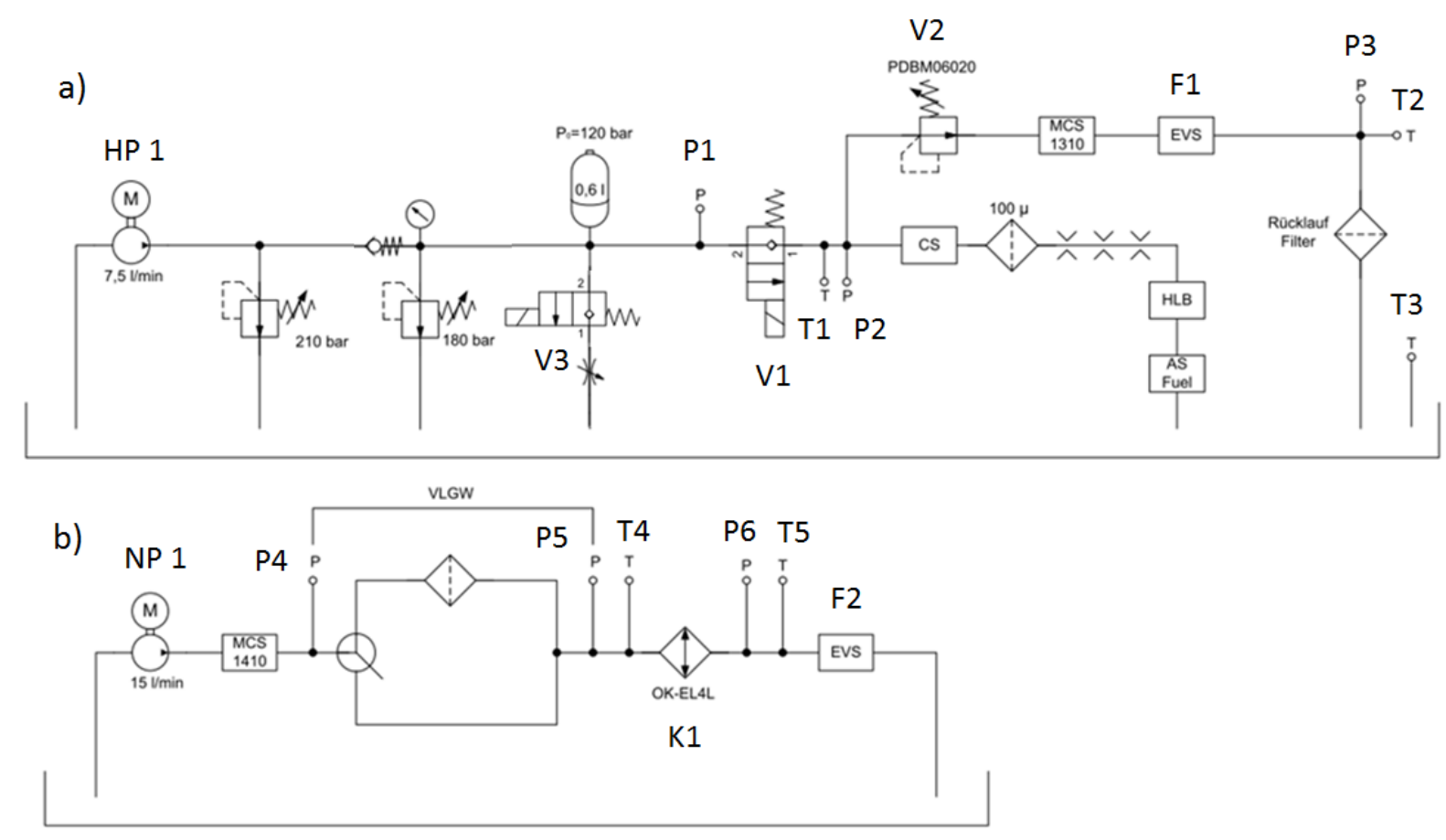

Bild 2: Schaltplan Hydraulikaggregat mit Arbeitskreislauf (a) und Sekundärkreislauf (b). Ziel: Überwachung der Hauptpumpe HP1, des Wegeventils V1 und des Kühlers K1. Sensoren: Druck (P), Temperatur (T), Fluss (F), zusätzlich Vibrations- und Leistungsmessung an HP1 und verschiedene Sensoren zur Ölqualitäts- und Partikelkontaminationsüberwachung (CS, MCS, HLB, AS Fuel)

Tabelle 1: Fehlerszenarien: Komponentenzustände und deren Erzeugung

\begin{tabular}{|c|c|c|c|}
\hline Komponente & Fehlerzustand & Steuerung & Möglicher Bereich \\
\hline Kühler K1 & Kühlwirkung & Gepulster Betrieb des Lüfters & Stufenlos $0 \%-100 \%$ \\
\hline Pum & e & arallels & $\begin{array}{l}\text { Geschlossen, offen mit } \\
\text { Blende } 3 \times 0,2 \mathrm{~mm} \text { und } 3 \times \\
0,25 \mathrm{~mm} \text {, komplett offen }\end{array}$ \\
\hline Ventil V1 & Schaltverzögerung & Ansteuerstrom & Stufenlos $0-$ \\
\hline \multicolumn{4}{|c|}{$\begin{array}{l}\text { Das Hydraulikaggregat wurde dahingehend modifiziert, dass eine gezielte Ver- } \\
\text { schlechterung von Komponentenzuständen reversibel steuerbar ist (Tabelle 1). } \\
\text { Über eine LabVIEW Benutzeroberfläche können beliebige Verläufe konfigu- } \\
\text { riert und kombiniert werden, die automatisiert durchlaufen werden. Üblicher- } \\
\text { weise werden die verschiedenen Schadensfälle geordnet nach der Zeitkonstante } \\
\text { ihrer Symptome [7] ineinander verschachtelt (vgl. Bild 3b). Mit diesem Vorge- }\end{array}$} \\
\hline
\end{tabular}


hen lassen sich effizient Trainingsdaten mit beliebiger Permutation der Schadensfälle und -grade erstellen, wobei im Folgenden die Zustände der Komponenten Kühler K1, Pumpe HP1 und Ventil V1 betrachtet werden sollen.

\section{Charakterisierungsmessung und Ergebnis}

In einer Charakterisierungsmessung über 60 Stunden wurden bei gleichbleibendem Arbeitszyklus (Bild 3a) die drei Schadensfälle mit allen Permutationen durchlaufen und der Vorgang vier Mal wiederholt (Bild 3b). Hierbei wurden beim Lüfter des Kühlers die Tastgrade $100 \%$ (normal), $35 \%$ (poor) und $2 \%$ (near to failure) und beim Wegeventil $100 \%$ (normal), $86 \%$ (poor) und $73 \%$ (near to failure) des Nennstromes durchlaufen, wobei das Ventil bei $73 \%$ gerade noch zuverlässig schaltet. Die Pumpenleckage wurde durch das Zuschalten einer 3 x 0,25 mm Bypass-Blende erzeugt, die einen Leckagefluss von ca. 3,8 \% des Pumpenförderflusses von HP 1 bewirkt.

a)

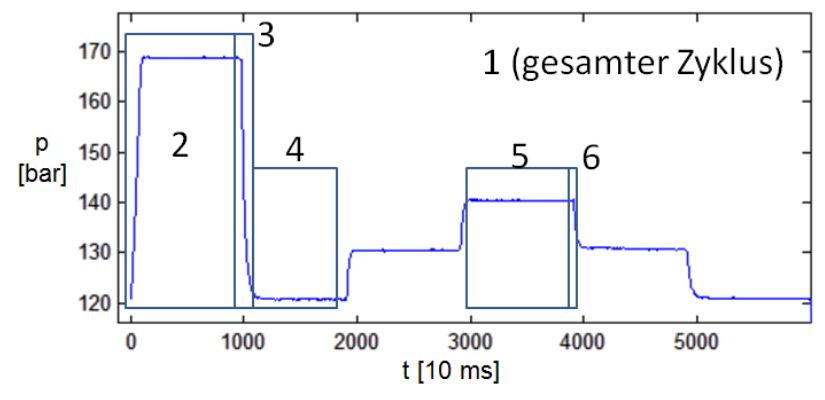

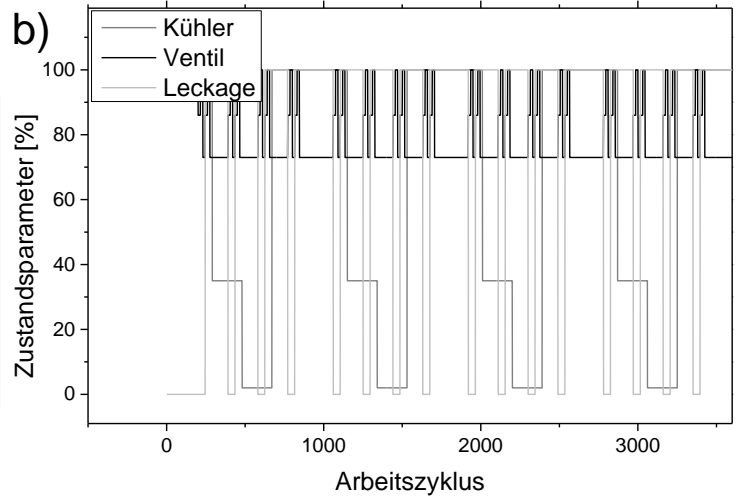

Bild 3 (a) Druckverlauf des Arbeitszyklus, gemessen von P1. Untersuchte Bereiche: (1) gesamter Zyklus, (2) Last getrennt, Druckbegrenzung aktiv, (3) Ventilschaltvorgang, (4) Last aktiv bei konstant niedrigem Druck, (5) Last aktiv bei konstant hohem Druck, (6) Druckabfall nach Lastsprung (b) Verlauf der Schadenssollwerte während der Charakterisierungsmessung

In Tabelle 2 sind die schadensspezifisch höchstkorrelierten Merkmale aufgeführt, die aus einem Pool von 336 automatisch generierten Merkmalen ausgewählt wurden. Die höchste Korrelation $(>0,9)$ besitzen die Merkmale für die 
Kühlerüberwachung, während die Merkmale zur Pumpenleckage den betragskleinsten Korrelationskoeffizienten $(<0,75)$ aufweisen.

Tabelle 2: Signifikanteste Merkmale (Sensoren, Funktionen (Mittelwert MW, Standardabweichung SA, Steigung ST) und Bereiche im Arbeitszyklus, vgl. Bild 3a) zur Überwachung der Komponenten und deren Korrelationskoeffizientenbetrag $|r|$

\begin{tabular}{lllll} 
Komp. & Sensor & Fkt. & Bereich & $|\mathrm{r}|$ \\
\hline \hline Kühler & Rel. Temperatur-Differenz & MW & $1,2,3,4,5,6$ & $0,94-0,95$ \\
K1 & (virtuell, mit T4, T5, $\mathrm{T}_{\text {amb }}$ ) & & & \\
& Druck vor Filter (P4) & MW & $1,2,3,4,5,6$ & 0,91 \\
& Druck Kühlerausgang (P6) & MW & $1,2,4,5$ & 0,91 \\
& Druck Kühlereingang (P5) & MW & $1,2,4,5$ & 0,91 \\
\hline Ventil & Druck nach Ventil (P2) & MW & 2,3 & $0,93-0,95$ \\
V1 & Druck vor Ventil (P1) & MW & 3 & 0,88 \\
& Druck nach Ventil (P2) & SA & 2 & 0,88 \\
& Druck am Rücklauffilt. (P3) & MW & 3 & 0,87 \\
& Fluss (F1) & MW & 3 & 0,86 \\
& Druck nach Ventil (P2) & ST & 3 & 0,85 \\
\hline Pumpe & Fluss (F1) & MW & $5 ; 4 ; 1$ & 0,$75 ; 0,6 ; 0,6$ \\
HP1 & Wirkungsgrad (virtuell, mit P2, F1, el. & MW & $6 ; 1 ; 5$ & 0,$75 ; 0,6 ; 0,59$ \\
& Leistung) & & & \\
& Fluss (F1) & SA & 1 & 0,64 \\
& Druck am Rücklauffilt. (P3) & SA & 1,5 & 0,47
\end{tabular}

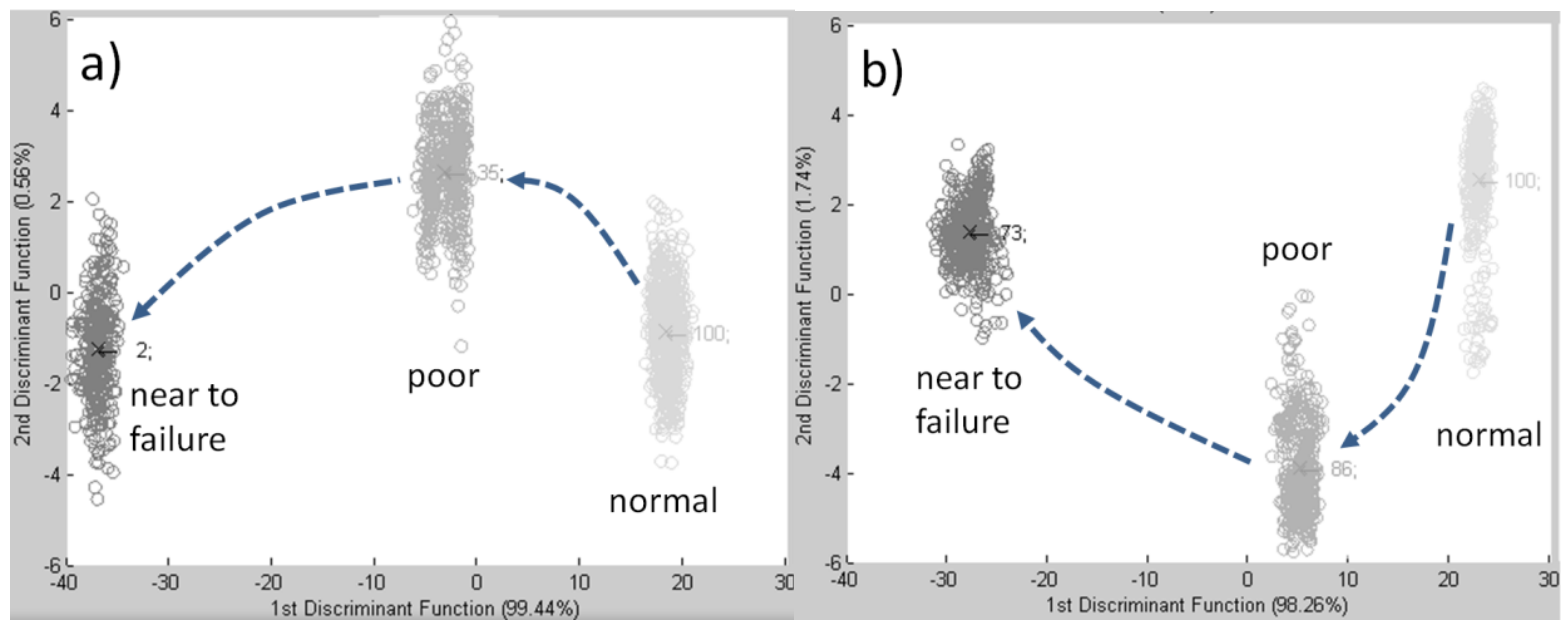

Bild 4: (a) Transformierte Trainingsdaten Kühlerzustand und (b) Ventilzustand 

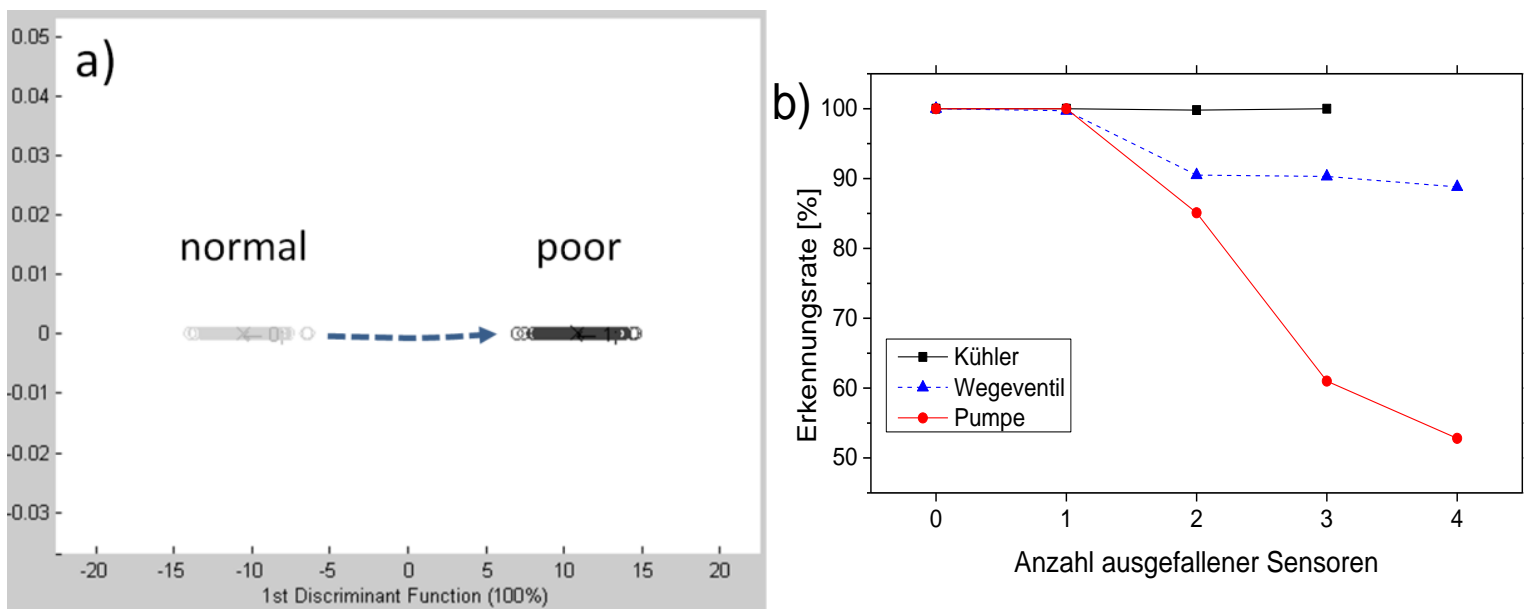

Bild 5: (a) Transformierte Trainingsdaten Pumpenzustand, (b) Erkennungsrate in Abhängigkeit der Anzahl ausgefallener signifikanter Sensoren unter Verwendung der Leave-One-Out-Cross Validation (LOOCV) mit k-nächste-NachbarnKlassifikator $(\mathrm{k}=3)$

Die Ergebnisse der LDA für die Schadensfälle sind in Bild 4 und 5a dargestellt. Es lässt sich eine vollständige Klassentrennung der unterschiedlichen Komponentenzustände erzielen, wobei die erste Diskriminanzfunktion jeweils als quantitative Größe zur Zustandsbeschreibung geeignet ist. Durch die Überlagerung der Schadensfälle kann die LDA Quereinflüsse (vor allem der Öltemperatur) gezielt unterdrücken, was der Robustheit der Zustandsüberwachung zugute kommt. In Bild 5b ist die Erkennungsrate in Abhängigkeit der Anzahl ausgefallener Sensoren dargestellt (Ausfallreihenfolge mit absteigendem Korrelationskoeffizient). Hierbei zeigt sich, dass der Ausfall des höchstkorrelierten Sensors in allen drei Fällen nach erneutem Training vollständig von den übrigen Sensoren kompensiert werden kann. Die Erkennungsrate verschlechtert sich erst beim gleichzeitigen Ausfall von zwei Sensoren bei der Wegeventil- und Pumpenüberwachung deutlich von $100 \%$ auf 90,1\% bzw. 85,1\%, während die Kühlerüberwachung in diesem Fall auf konstant hohem Niveau verbleibt.

\section{Fazit}

In diesem Beitrag wurde ein Verfahren für das Condition Monitoring von Hydraulikanlagen vorgeschlagen und erfolgreich validiert, das mittels Korrelationsanalyse automatisiert geeignete Merkmale zur Überwachung einzelner Kompo- 
nenten aus den Sensorrohdaten extrahiert und diese mittels linearer Diskriminanzanalyse auf eine quantitative Größe reduziert, welche ein Maß für den jeweiligen Zustand ist. Des Weiteren konnte gezeigt werden, dass das CMSystem in der Lage ist, den Ausfall des jeweils signifikantesten Sensors vollständig zu kompensieren.

\section{Danksagung}

Dieses Projekt wurde vom Programm Förderung von Entwicklung, Forschung und Innovation im Saarland (EFI) gefördert und von HYDAC Filter Systems $\mathrm{GmbH}$ (Sulzbach / Saarland) finanziert.

\section{Literatur}

[1] F. Marquez et al., Condition monitoring of wind turbines: Techniques and methods, Renewable Energy 46, 169-178, Elsevier, 2012

[2] Pierre Tchakoua et al., Wind Turbine Condition Monitoring: State-of-theArt Review, New Trends, and Future Challenges, Energies 2014, 7, 25952630, doi:10.3390/en7042595

[3] Ahemd El-Betar et al., Fault Diagnosis of a Hydraulic Power System Using an Artificial Neural Network, JKAU: Eng. Sci., Vol. 17 No. 1, pp: 117 137

[4] Xuexia Liu et al., Study on Knowledge -based Intelligent Fault Diagnosis of Hydraulic System, TELKOMNIKA, Vol.10, No.8, December 2012, pp. 2041 2046, e-ISSN: 2087-278X

[5] A. Guenel, A. Meshram, T. Bley, A. Schütze, M. Klusch: Statistical and Semantic Multisensor Data Evaluation for Fluid Condition Monitoring in Wind Turbines. Proc. 16th International Conference on Sensors and Measurement Technology (SENSOR), Nuremberg, Germany, 2013

[6] J.P. Marques de Sá, Pattern Recognition - Concepts, Methods and Applications, Springer Verlag, Berlin, 2001

[7] International Standard ISO 13372 - Condition monitoring and diagnosis of machines - Vocabulary, 2004 